\title{
Removal of Foreign Body Aided with Various Navigation Modalities: A Case Report and Literature Review
}

\author{
Peng Li, Hongyu Guan, Jing Song, Dahong Huang* \\ Foshan Stomatology Hospital, Foshan University, Foshan, P. R. China
}

Email address:

fshuangdh@126.com (Dahong Huang)

${ }^{*}$ Corresponding author

\section{To cite this article:}

Peng Li, Hongyu Guan, Jing Song, Dahong Huang. Removal of Foreign Body Aided with Various Navigation Modalities: A Case Report and Literature Review. Journal of Surgery. Vol. 9, No. 2, 2021, pp. 86-92. doi: 10.11648/j.js.20210902.19

Received: February 17, 2021; Accepted: March 9, 2021; Published: April 13, 2021

\begin{abstract}
Background: Computerized surgical navigation (CSN) has many potential applications in foreign body (FB) removal. Case report: While playing, a 14-year-old girl was accidentally shot in the facial area with a shotgun. Removal of the superficial shots and of the left eyeball was performed at a local hospital. She was transferred to the West China Hospital of Stomatology (Sichuan, China) on the third day after the accident due to difficulties in removing the remaining shots. Except for left eye vision loss, there was no abnormal physical or laboratory test. Spiral computed tomography (CT) scan of the head was performed and data were imported into the iplan software of the VectorVision ${ }^{2}$ navigation system. A dynamic reference frame was fixed to the skull and another to the forceps. Eight shots were successfully removed. One shot could not be removed because of significant shifting. At 1 month, the incisions were successfully healed and there were no symptoms or signs. Conclusion: The case presented here and the relevant literature about CSN indicates that CSN is a valuable modality for the removal of FBs, not only in the craniomaxillofacial area, but anywhere in the body.
\end{abstract}

Keywords: Computerized Surgical Navigation, Foreign Body, Computer-Aided Design, Maxillofacial Surgery

\section{Introduction}

The craniomaxillofacial area is an area with a large number of complex and delicate anatomic structures. Injuries to those structures may result in mortality or important morbidity with significantly affected quality of life $[1,2]$. Craniomaxillofacial injury may be the result of low-energy impact (e.g., falls or fighting), high-energy trauma (e.g., car accidents and firearms), or iatrogenic injury (e.g., broken needle during dental surgery). Wound management often requires the removal of foreign bodies (FBs) embedded among the delicate structures, and those structures are at risk of being further damaged during removal surgery [3]. Leaving those FBs in place is usually the last option because of the possibility of morbidity, abscess, and worsening of the condition [4].

High-energy traumas often involve the craniomaxillofacial area and often result in dismal outcomes [3, 5]. Shotgun wounds are particularly complex to manage because of the large numbers of small metal balls imbedded at various depth and over a large body area $[6,7]$. If not fatal, craniomaxillofacial shotgun wounds are particularly difficult to manage due to the risk of further injury when removing the FBs $[8,9]$

Historically, the surgical approach for FB removal was limited to the exploration of the wounds by palpation with or without visual guidance based X-ray or computed tomography (CT); this approach is greatly limited by the fact that imaging and the surgeon are too distinct and non-coordinated entities, which could result in further iatrogenic injury, migration of the FBs, or failure to remove the FBs [4, 10]. Computerized surgical navigation (CSN) is developing enthusiastically and has many potential applications in many fields such as trauma or cancer reconstruction surgery, orthognathic surgery, skull base surgery, and FB removal $[11,12]$. Importantly, it allows the "fusion" of the surgical gesture with imaging data, improving accuracy, especially in the presence of delicate structures. Many studies have reported the CSN-guided removal of FB from the human body, especially in the complex and delicate craniomaxillofacial area [13-32]. CSN is helpful in determining the location of FB, determining the optimal surgical approach, minimizing iatrogenic damage, and reducing the operation time. With the development of CSN, the operation process is becoming more convenient and accurate. 
In this paper, we present one case of shotgun buckshot removal from the maxillofacial area, as well as a review of the literature and a discussion about the standard strategy for CSN-guided removal of FBs in the craniomaxillofacial area.

\section{Case Presentation}

This study was approved by the ethics committee of the West China Hospital of Stomatology. Informed consent for publication was obtained from the patient.

While playing, a 14-year-old girl was accidentally shot in the facial area with a shotgun. She was admitted to a local hospital as soon as possible after the accident. At this local hospital, removal of the superficial shots and of the left eyeball was performed. Due to the difficulties in removing the deeper shots, the patient was transferred to the oral and maxillofacial surgery department of the West China Hospital of Stomatology (Sichuan, China) on the third day after the accident. Physical examination showed multiple buckshot wounds located on the left upper face and forehead. The left eyeball had been removed (Figure 1). A panoramic radiograph indicated multiple small spheres with high-density in the facial area. A CSN operation was prepared for removing these FBs because of the risk of chronic pain if they were left in place.

At admission, the patient was fully conscious and did not have any neurological deficits. Imaging showed no damage to the brain parenchyma. Except for left eye vision loss, there was no abnormal physical or laboratory test. Spiral computed tomography (CT) scan of the head was performed. The CT data, with $0.625-\mathrm{mm}$ slice thickness, were imported into the iplan software of the VectorVision ${ }^{2}$ navigation system (BrainLAB, Feldkirchen, Germany). In the iplan software, the buckshots could be clearly viewed. A virtual three-dimensional (3-D) model was built to show the nine buckshots of $5 \mathrm{~mm}$ in diameter.

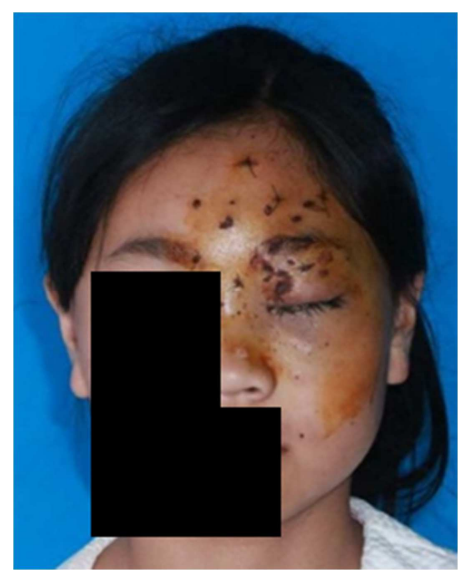

Figure 1. The patient who received a gunshot wound after emergency treatments at the local hospital and before navigation-guided surgery.
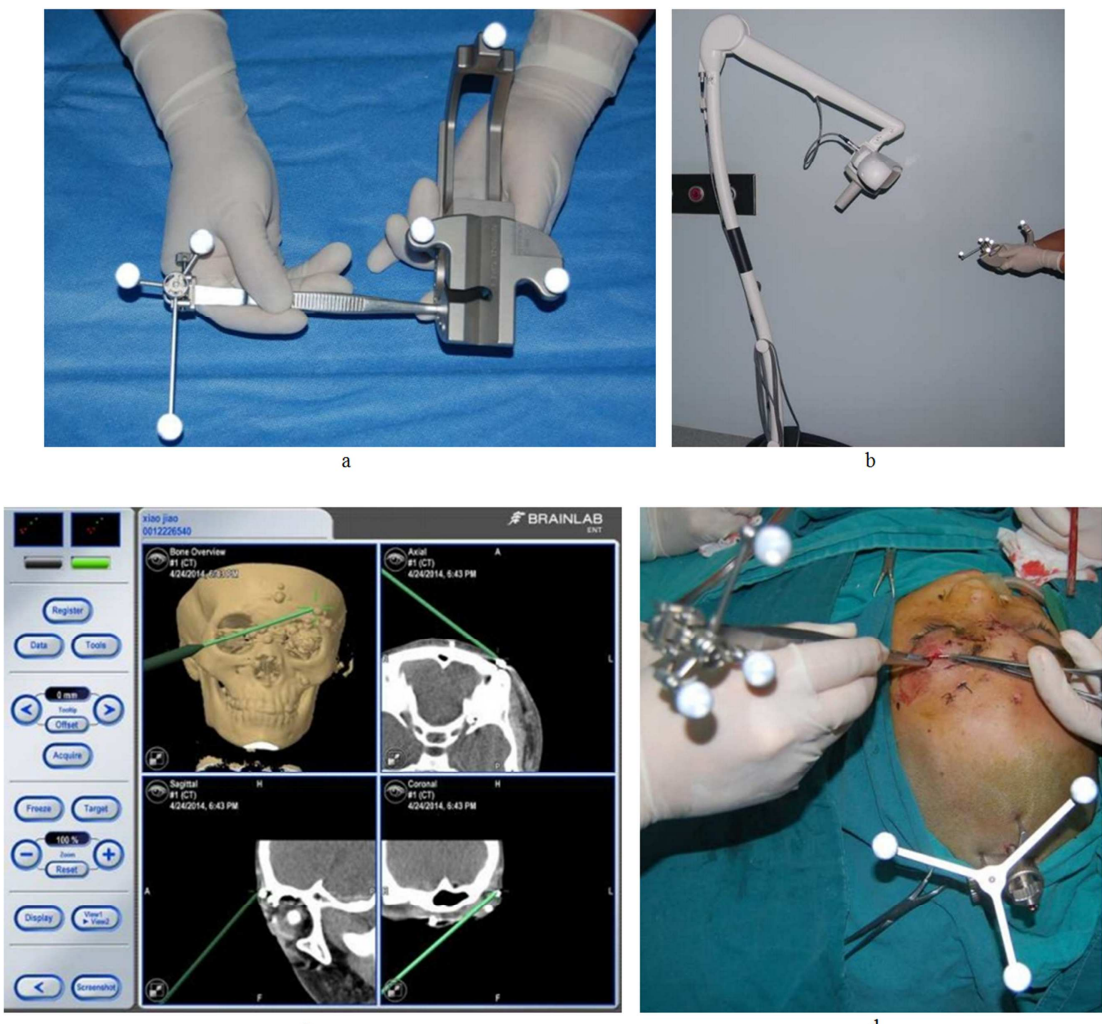

Figure 2. Procedure of the navigation-guided surgery. (a) The forceps clamped with a dynamic reference frame inserting into the calibration hole of the registration instrument. (b) The forceps with tracking camera for registration. (c) Real-time screen capture of the BrainLAB system indicating the navigation pointer arriving at one buckshot. (d) Removal of one buckshot directly using the calibrated forceps.

During surgery, the dynamic reference frame was rigidly fixed on the patient's skull with one monocortical screw under general anesthesia. Face scanning was performed with the Z-touch laser scanner from the BrainLAB system 
(Feldkirchen, Germany) for surface registration. A dynamic reference frame was clamped at the end of one forceps. The forceps was inserted into the calibration hole of the registration instrument, and it was registered by the tracking camera. After registration, the tip position and orientation of the forceps could be viewed on the flat-panel monitor in real time. The location accuracy (within $1.5 \mathrm{~mm}$ ) was verified using the navigation pointer. With the guidance of the BrainLAB system, the registered forceps, which was tracked by the navigation system, could directly detect the exact anatomical site of each buckshot in the facial area. Eventually, eight buckshots were clamped and removed successfully through the original wound path (Figure 2a-d). The whole surgical procedure, including the installation and registration of the navigation system, took approximately $40 \mathrm{~min}$. Intraoperative blood loss was approximately $50 \mathrm{ml}$. Unfortunately, even after multiple tries, one buckshot located in the left cheek could not be removed.

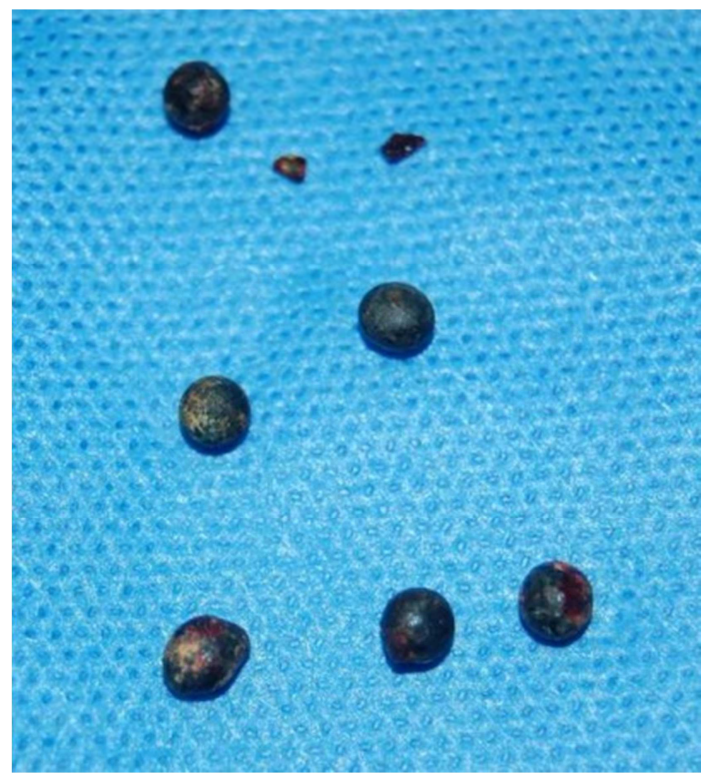

a

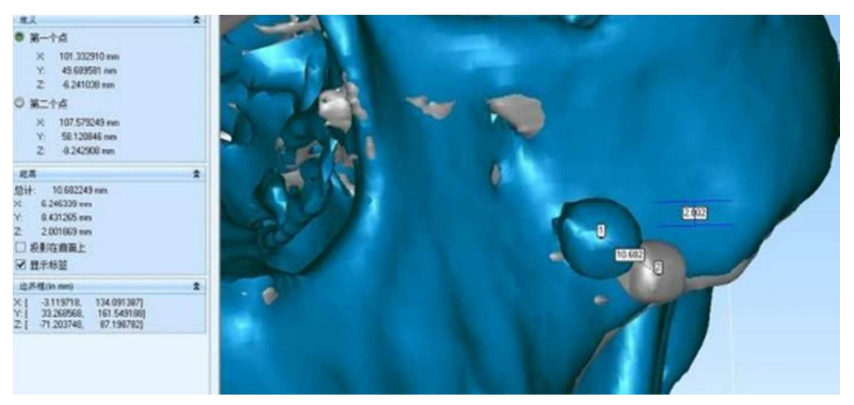

b

Figure 3. (a) Eight removed buckshots. (b) Image fusion of the preoperative and postoperative 3-D models.

She was discharged from the hospital 2 days later without complications. A cone-beam CT scan of the head was done and a 3-D model was established. Through image fusion of the preoperative and postoperative 3-D models, it was found that the remaining buckshot shifted by about $10 \mathrm{~mm}$ posterior and inferior to its original position (Figure 3a-b), probably because of the movements of the mandible and facial muscles, and because of palpation and movements from the forceps. At 1 month of follow-up, the incisions in the face were healed. The patient showed no clinical symptom. Afterwards, the girl's condition improved quickly and she recovered uneventfully.

\section{Literature Review}

A systematic literature search from electronic databases (MEDLINE (PubMed), the Cochrane library, EMBASE, Web of Science, and Google) was carried out using the medical subject heading (MeSH) terms "foreign body" and "navigation" or "surgical navigation". More than 50 relevant studies in English published between 2000 and 2019 were found and reviewed. The inclusion criteria were: 1) clinical case report; and 2) FB removal with CSN. The exclusion criterion was FB removal surgery using other image-guidance technique. If the title was considered relevant, the abstract was looked through with regard to inclusion or exclusion criteria. According to the above inclusion criteria, a hand search of the relevant journals was also performed. Finally, 20 articles were included and reviewed. The relevant data from the 20 articles were organized in spreadsheets and the following information was extracted: Reference, number of patients, FB type, location, CT slice thickness, navigation system, assistant 3-22 method, and intraoperative time (Table 1).

It can be concluded from Table 1 that the vast majority of the published papers are case reports or studies of no more than five patients. Most of FBs were broken needles, metal fragments or projectiles, and located in the craniofacial area, which is a complex anatomic zone and has numerous vital or delicate structures. Only four papers reported about the removal of firearm projectiles and shrapnel [16, 24, 27, 32]. With the help of CSN, all FBs in the 20 papers were successfully located and removed, with or without assistant method. None of the 20 articles investigated the accuracy and intra-operative precision of CSN, but is have already been proven to be $<1 \mathrm{~mm}$ and $1-2 \mathrm{~mm}$, respectively [12]. Few of the 20 papers took note of preoperative planning and intraoperative time in details. As the CSN technique has been proved as a valuable tool, surgeons' and patients' time and costs should be considered for this kind of research in the future. In addition, how to detect non-metallic FBs accurately should be considered.

\section{Discussion}

We present a case of an accidental shotgun discharge to the craniomaxillofacial area of a 14-year-old girl. Removal of the superficial shots and of the left eyeball was performed at a local hospital. She was transferred to a tertiary center on the third day after the accident due to difficulties in removing the remaining FBs using conventional methods. Except for left eye vision loss, there was no abnormal physical or laboratory test. Successful 3D modeling and navigation was performed 
using spiral CT data imported into the iplan software of the VectorVision $^{2}$ navigation system. Finally, eight shots were successfully removed, but one shot could not be removed because of significant shifting. At 1 month, the incisions were successfully healed and there were no symptoms or signs from the remaining $\mathrm{FB}$.

Table 1. Literature review of the use of CSN for FB removal.

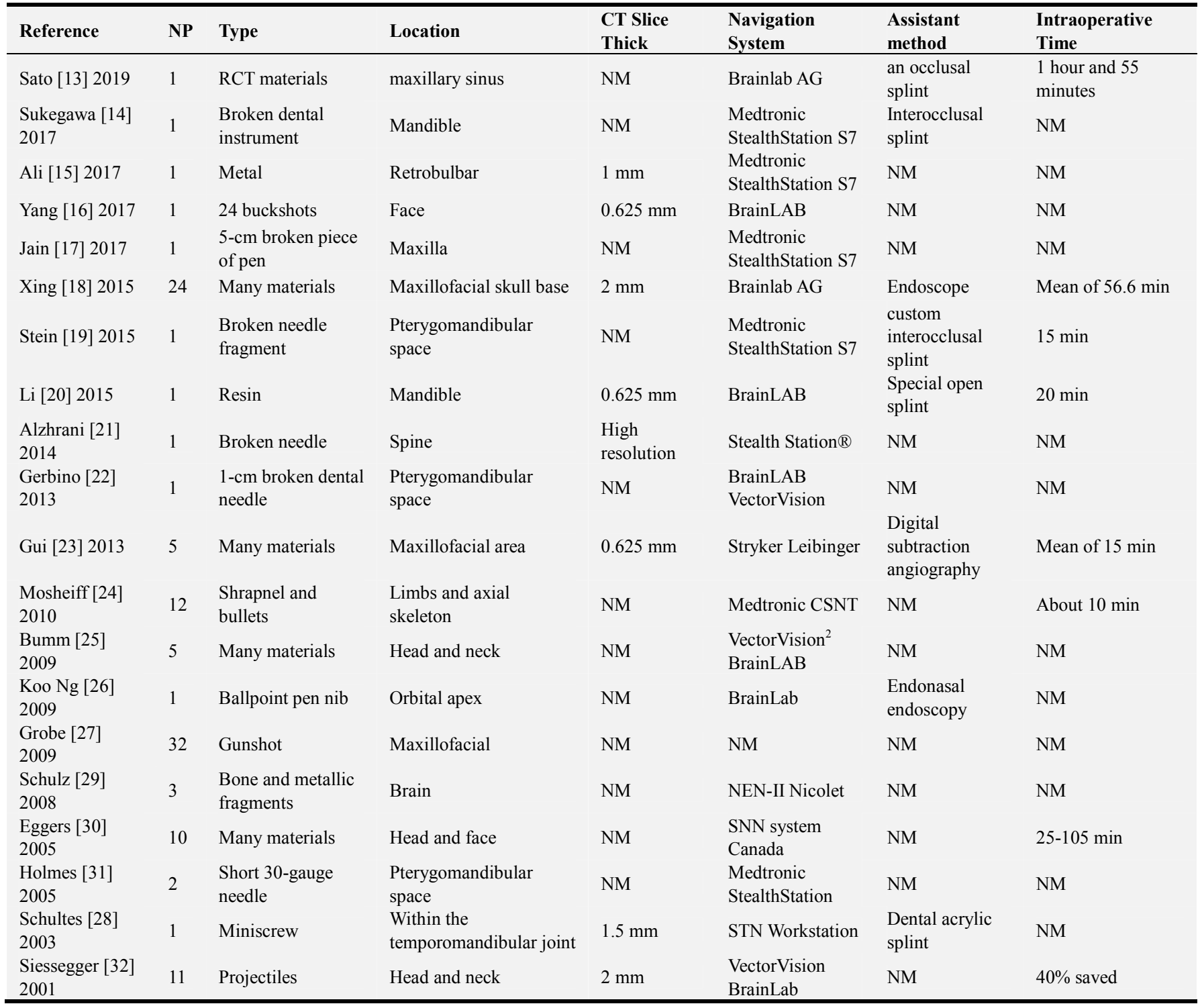

$\mathrm{NP}=$ number of patients; $\mathrm{NM}=$ no mentioned.

Traditional removal of FBs usually involves direct exploration of the wounds or first visualizing the FBs using traditional imaging methods such as X-ray, C-arm fluoroscopy, ultrasound, and CT, followed by removal of the FBs based on imaging. The first approach is a blind approach, while the second is a semi-blind approach. Indeed, even if the FBs can be seen on the images, how to translate the images to the actual patient requires skill and experience [4, 10]. Both approaches have a high potential of resulting in iatrogenic injuries or failures to remove the FBs, leading to additional morbidity [4, 10]. Using $\mathrm{C}$-arm X-ray, $\mathrm{He}$ et al. [33] introduced a novel navigation system for the removal of metallic FBs retained in soft tissue: the target spot is displayed on the screen and a laser is used on the skin surface to indicate the most optimal path. Ultrasound can provide real-time imaging of the FB, but the probe has to maintained on the skin with some pressure, which impedes surgery and can cause shifting of the FB.

A possible solution to improve the removal rate of difficult to reach FBs is CSN-guided surgery, which is less invasive, is more precise, and takes a shorter time than exploratory traditional surgery, and has therefore lower operative costs in terms of staff time. On the other hand, the main drawback is the need for expensive specialized medical equipment that is available only in a few centers.

The first consideration about to use CSN or not is whether the FB should be removed [34]. Gui et al. [23] suggested that all FBs leading to clinical symptoms or located nearby vital 
structures should be removed to prevent complications. This is particularly crucial in the craniomaxillofacial area because of the delicate anatomical structures and of the constant and complex movements of the muscles that can cause further injuries and morbidity [4]. Based on our experience, the following conditions are recommended indications for CSN in FB removal surgery: 1) need for minimally invasive procedures; 2) presence of multiple FBs; 3) FB nearby some vital organs or structures; and 4) failure of previous attempts with conventional techniques. The second consideration of $\mathrm{CSN}$ is to determine the optimal timing to remove these FBs, and this point is still controversial [35]. For navigation-guided surgery, image data acquired preoperatively should accurately represent the structure of the tissue during operation. Fibrous connective tissue will eventually develop around each FB, which may limit the shifting of the FB and may be beneficial to improve the surgical success. Indeed, even if the elapsed time between imaging and surgery is kept as short as possible, simply moving the patient from the scanner to the surgical bed can shift some FBs that are not fixed in place. Since the soft tissues are not physically stationary, it is important to carry out the surgery immediately after the preoperative CT scans for ensuring the accuracy of CSN, and to limit the movements of the patient as much as possible. In addition, the inflammatory reaction of the soft tissue in the early stage after trauma will also affect the accuracy of image and can hide some minute FBs. Therefore, a wait of at least 1 week may be recommended before removal.

A large number of systematic reviews and retrospective studies have investigated recent developments of CSN in the field of FB removal surgery, and demonstrated promising results [11, 23, 34-37]. Well-designed prospective study could provide objective evaluation, but such studies are especially challenging due to several confounding factors such as various locations of the FB, various FB materials, and the physical condition of the patients. Kaviani et al. [38] conducted a blind trial, in which five test specimens of different materials (plastic, glass, wood, metal, and stone) were inserted into the craniomaxillofacial area of ten body donors; then, removal surgery was performed with or without the help of CSN. Their results suggest that current $\mathrm{CSN}$ is an effective tool for $\mathrm{FB}$ removal in high-density areas such as the craniomaxillofacial area.

A customized guide bar could been designed to facilitate removal of a FB. Xing et al. [39] discussed a special 3-D locator and magnetic forceps for the removal of metallic FBs in soft tissue, including hand or foot, upper extremity, lower limb, hip, chest or and abdomen; $99.5 \%$ of 7390 patients were successfully operated within a mean of $5 \mathrm{~min}$. Ma et al. [35] reported a case of removal of two pieces of glass in the right side of face with the help of facial soft-supported digital guide, despite the cost and extra time needed for preparation before the operation. Nevertheless, optimization of the process is required due to the wide variety of possible sites for FBs.

Taken together, the case presented here and the relevant literature about CSN indicates that $\mathrm{CSN}$ is a valuable modality for the removal of FBs, not only in the craniomaxillofacial area, but anywhere in the body. This paper adds some systematic and conclusive knowledge to the CSN literature.

\section{Conclusion}

Computerized surgical navigation (CSN) has many potential applications in foreign body (FB) removal. The case presented here illustrated the process of removal of FBs in the craniomaxillofacial area in detail, and the relevant literature about CSN indicates that it is a valuable modality for many kinds of surgery in other parts of the body.

\section{Funding}

This work was supported by the Natural Science Foundation of Guangdong Province (No. 2018A030313847) and the High-Level Talent Start-Up Research Project of Foshan University (Gg 07002).

\section{Compliance with Ethical Standards}

This study was approved by the ethics committee of the West China Hospital of Stomatology. Informed consent for publication was obtained from the patient.

\section{Conflicts of Interest}

The authors declare that they have no competing interests.

\section{Acknowledgements}

I gratefully acknowledge to all those who helped me during the writing of this thesis, especially my supervisors, Dr. WEIDONG TIAN and Dr. WEI TANG, who have offered me valuable suggestions in the academic studies.

\section{References}

[1] DeLong MR, Gandolfi BM, Barr ML, Datta N, Willson TD, et al. Intraoperative Image-Guided Navigation in Craniofacial Surgery: Review and Grading of the Current Literature. J Craniofac Surg 2019; 30: 465-472.

[2] Shah I, Gadkaree SK, Tollefson TT, Shaye DA. Update on the management of craniomaxillofacial trauma in low-resource settings. Curr Opin Otolaryngol Head Neck Surg 2019; 27: 274-279.

[3] Haug RH, Morgan JP Etiology, Distribution, and Classification of Traumatic Craniomaxillofacial Deformities and Defects. In: Greenberg A, Schmelzeisen R, editors. Craniomaxillofacial Reconstructive and Corrective Bone Surgery. New York: Springer. 2019.

[4] Marcus JR, Erdmann D, Rodriguez ED (2012) Essentials of Craniomaxillofacial Trauma. St Louis: Quality Medical Publishing, Inc. 
[5] Vaca E E, Bellamy J L, Sinno S, et al. Management of High-energy Avulsive Ballistic Facial Injury [J]. Plastic and Reconstructive Surgery - Global Open, 2018, 6 (3): e1693.

[6] Rhee PM, Moore EE, Joseph B, Tang A, Pandit V, et al. Gunshot wounds: A review of ballistics, bullets, weapons, and myths. J Trauma Acute Care Surg 2016; 80: 853-867.

[7] Schellenberg M, Inaba K, Heindel P, et al. The diagnostic dilemma of shotgun injuries [J]. European Journal of Trauma and Emergency Surgery, 2019 (6).

[8] Kaufman Y, Cole P, Hollier L Contemporary issues in facial gunshot wound management. J Craniofac Surg 2008; 19: 421-427.

[9] Kaufman Y, Cole P, Hollier LH, Jr. Facial gunshot wounds: trends in management. Craniomaxillofac Trauma Reconstr 2009; 2: 85-90.

[10] Voss JO, Thieme N, Doll C, Hartwig S, Adolphs N, et al. Penetrating Foreign Bodies in Head and Neck Trauma: A Surgical Challenge. Craniomaxillofac Trauma Reconstr 2018; 11: 172-182.

[11] Azarmehr I, Stokbro K, Bell RB, Thygesen T Surgical Navigation: A Systematic Review of Indications, Treatments, and Outcomes in Oral and Maxillofacial Surgery. J Oral Maxillofac Surg 2017; 75: 1987-2005.

[12] Dai J, Wu J, Wang X, Yang X, Wu Y, et al. An excellent navigation system and experience in craniomaxillofacial navigation surgery: a double-center study. Sci Rep 2016; 6: 28242.

[13] Sato H, Shiogama S, Narihira K Removal of small foreign bodies from the maxillary sinus using $\mathrm{CT}$ image-guided surgical navigation and rapid paired-point registration and calibration with reference markers on an occlusal splint. Oral Maxillofa Surg Cases 5. 2019.

[14] Sukegawa S, Kanno T, Shibata A, Matsumoto K, Sukegawa-Takahashi $\mathrm{Y}$, et al. Use of an intraoperative navigation system for retrieving a broken dental instrument in the mandible: a case report. J Med Case Rep 2017 11: 14.

[15] Ali MJ, Naik MN, Kaliki S, Dave TV, Dendukuri G Interactive navigation-guided ophthalmic plastic surgery: the techniques and utility of 3-dimensional navigation. Can J Ophthalmol 2017; 52: 250-257.

[16] Yang CY, Yang RT, He SG, Li Z Removal of a large number of foreign bodies in the maxillofacial region with navigation system. Dent Traumatol 2017; 33: 230-234.

[17] Jain A, Gupta G, Grover M Removal of an Unusual Neglected Foreign Body in Infratemporal Region Using Navigation. J Craniofac Surg 2017; 28: e219-e221.

[18] Xing L, Duan Y, Zhu F, Shen M, Jia T, et al. Computed tomography navigation combined with endoscope guidance for the removal of projectiles in the maxillofacial area: a study of 24 patients. Int J Oral Maxillofac Surg 2015; 44: 322-328.

[19] Stein KM Use of Intraoperative Navigation for Minimally Invasive Retrieval of a Broken Dental Needle. J Oral Maxillofac Surg 2015; 73: 1911-1916.

[20] Li P, Li Z, Tian W, Tang W A strategy for removal of foreign body in mandible with navigation system. Int J Oral Maxillofac Surg 2015; 44: 885-888.
[21] Alzhrani GA, Alturki AY, Almusrea KN Removal of lumber spine foreign body using minimal access system with navigation. Asian J Neurosurg 2015; 10: 108-110.

[22] Gerbino G, Zavattero E, Berrone M, Berrone S Management of needle breakage using intraoperative navigation following inferior alveolar nerve block. J Oral Maxillofac Surg 2013; 71 : $1819-1824$

[23] Gui H, Yang H, Shen SG, Xu B, Zhang S, et al. Image-guided surgical navigation for removal of foreign bodies in the deep maxillofacial region. J Oral Maxillofac Surg 2013; 71: 1563-1571.

[24] Mosheiff R, Weil Y, Khoury A, Liebergall M The use of computerized navigation in the treatment of gunshot and shrapnel injury. Comput Aided Surg 2004; 9: 39-43.

[25] Bumm K, Bohr C, Bozzato A, Wurm J Soft tissue navigation and image-guided removal of foreign bodies in head and neck surgery. HNO 2009; 57: 1016-1022.

[26] Koo Ng NK, Jaberoo MC, Pulido M, Olver JM, Saleh HA Image guidance removal of a foreign body in the orbital apex. Orbit 2009; 28: 404-407.

[27] Grobe A, Weber C, Schmelzle R, Heiland M, Klatt J, et al. The use of navigation (BrainLAB Vectorvision (2)) and intraoperative 3D imaging system (Siemens Arcadis Orbic 3D) in the treatment of gunshot wounds of the maxillofacial region. Oral Maxillofac Surg 2009; 13: 153-158.

[28] Schultes G, Zimmermann V, Feichtinger M, Gaggl A, Karcher H Removal of osteosynthesis material by minimally invasive surgery based on 3-dimensional computed tomography-guided navigation. J Oral Maxillofac Surg 2003; 61: 401-405.

[29] Schulz C, Woerner U, Luelsdorf P Image-guided neurosurgery for secondary operative removal of projectiles after missile injury of the brain. Surg Neurol 2008; 69: 364-368.

[30] Eggers G, Haag C, Hassfeld S Image-guided removal of foreign bodies. Br J Oral Maxillofac Surg 2005; 43: 404-409.

[31] Holmes PJ, Miller JR, Gutta R, Louis PJ Intraoperative imaging techniques: a guide to retrieval of foreign bodies. Oral Surg Oral Med Oral Pathol Oral Radiol Endod 2005; 100: 614-618.

[32] Siessegger M, Mischkowski RA, Schneider BT, Krug B, Klesper B, et al. Image guided surgical navigation for removal of foreign bodies in the head and neck. J Craniomaxillofac Surg 2001; 29: 321-325.

[33] He B, Xu C, Mao Y, Mao J, Shen L, et al. A novel navigation system to guide metallic foreign body extraction. Int J Comput Assist Radiol Surg 2016; 11: 2105-2110.

[34] Ji Y, Jiang H, Wan L, Yuan H Effect of Navigation System on Removal of Foreign Bodies in Head and Neck Surgery. J Craniofac Surg 2018; 29: e723-e726.

[35] Ma W, Wang LD, Liang Y, Li M Application of a digital guide in the removal of foreign body from the maxillofacial region. Br J Oral Maxillofac Surg 2019; 57: 708-709.

[36] Kumar V, Gour S, Chaube RK, Chaube RK, Gupta A, et al. Surgical Navigation in Oral and Maxillofacial Surgery: A Review. Clin Res Open Access 2018; 4: 1-6.

[37] Sukegawa S, Kanno T, Furuki Y Application of computer-assisted navigation systems in oral and maxillofacial surgery. Jpn Dent Sci Rev 2018; 54: 139-149. 
[38] Kaviani F, Javad Rashid R, Shahmoradi Z, Gholamian M Detection of foreign bodies by spiral computed tomography and cone beam computed tomography in maxillofacial regions. J Dent Res Dent Clin Dent Prospects 2014; 8: 166-171.
[39] Xing GF, Shi CW, Qian HX, Qin XJ Novel methods of removing metallic foreign body from human soft tissue: a report of 7390 cases. J Surg Res 2013; 183: 337-340. 